\title{
Type of setting for the inpatient adolescent with an eating disorder: Are specialized inpatient clinics a must or will the pediatric ward do?
}

\author{
Sinem Akgül ${ }^{1}$, Melis Pehlivantürk-Kızlkan¹, Sine Örs², Orhan Derman¹, Yasemin Düzçeker¹, \\ Nuray Kanbur ${ }^{1}$ \\ ${ }^{1}$ Division of Adolescent Medicine, Department of Pediatrics, ${ }^{2}$ Nutrition and Diet Unit, Hacettepe University Faculty of \\ Medicine, Ankara, Turkey. \\ E-mail: nuraykanbur@hotmail.com \\ Received: 8th December 2016, Accepted: 12th March 2017
}

SUMMARY: Akgül S, Pehlivantürk-Kızılkan M, Örs S, Derman O, Düzçeker Y, Kanbur N. Type of setting for the inpatient adolescent with an eating disorder: Are specialized inpatient clinics a must or will the pediatric ward do? Turk J Pediatr 2016; 58: 641-649.

There are a range of different services for treating adolescent eating disorders (ED) but there is no clinical consensus and a paucity of research indicating which type of treatment setting is the best. Although it would be ideal to have a specialized ward for these patients what happens when this is not possible? The aim of this study was to evaluate patients with ED hospitalized on a general pediatric ward. A retrospective chart review for 37 patients hospitalized for an ED and followed by a team consisting of an adolescent medicine specialist, a child and adolescent psychiatrist and a dietician on a pediatric ward were re-evaluated. Twenty-four $(64.9 \%)$ patients were diagnosed with anorexia nervosa (AN) restricting type, 8 (21.6\%) with anorexia nervosa binging purging type, $3(8.1 \%)$ with bulimia nervosa (BN) and $2(5.4 \%)$ with eating disorder otherwise not specified. The mean age at admission was $14.79 \pm 1.75$ years and $7(20 \%)$ were males. A majority were hospitalized due to medical instability. Mean period of time from admittance to medical stabilization was $6.04 \pm 4.79$ days. The mean period of admittance was $26.4 \pm 11.9$ days for AN and $23.7 \pm 15.03$ days for BN patients. The mean calorie intake of the AN group was $607 \pm 333 \mathrm{kcal}$ and 2,358 $\pm 605 \mathrm{kcal}$ at hospitalization and discharge, respectively. Hypophosphatemia occurred in 2 patients during refeeding. Mean total weight gained during the whole hospitalization period was $3,950 \pm 3,524 \mathrm{grs}$. This study shows that although not ideal, EDs can successfully be followed on general pediatric wards and could have implications at centers with no specialized wards.

Key words: admission, adolescents, clinical setting, eating disorder.

There are a few different treatment options for adolescent patients with an eating disorder (ED) such as; outpatient follow-up, day programs/ partial hospitalization, residential treatment centers and inpatient hospitalization ${ }^{1,2}$. Patients with ED's are more commonly hospitalized to treat medical complications resulting from the eating pathology, to treat comorbid psychiatric illnesses affecting treatment as an outpatient or for close surveillance of the patient due to risk of self-harm or complications that may occur due to compensatory behavior such as vomiting or laxative/diuretic abuse ${ }^{3}$.
In the current literature, criteria for hospitalization for adolescent patients with an ED are clear but are applied differently in various clinics in different countries depending on the health care systems ${ }^{4}$. Yet, no standard protocols are present for the initial stages of refeeding or the length of recovery and different models for hospitalization programs have been described. Although all programs provide stabilization of acute and severe medical complications, duration of inpatient management and approach to refeeding may change according to the center the patient 
receives treatment ${ }^{4}$. While some centers offer specialized inpatient care on wards specifically designated for EDs only, others like ours offer treatment on general pediatric wards designated for the adolescent age group but not specific for ED, where the ward staff have no psychiatric training. The treatment facilities available for these patients depend on many factors ranging from insurance issues to hospital policies ${ }^{5}$.

Although it would be ideal to have a separate ward for adolescents with ED, can these patients successfully be treated on pediatric wards? The aim of this paper was to explore the experience of an Adolescent Medicine Clinic with no specific inpatient unit for patients with ED that admit their patients with ED to a general pediatric ward for the adolescent age group and to discuss refeeding approaches and goals for discharge in this type of setting.

\section{Material and Methods}

\section{Study population}

This retrospective evaluation took place at Hacettepe University, Division of Adolescent Medicine. Between January 2010 and April 2016, 37 adolescents met criteria for admission to the general pediatric ward of our hospital. During January 2010- May 2013 diagnostic criteria of DSM-4 -TR was used whereas after May 2013 DSM-5 criteria was taken into account. The patients were initially evaluated at the Division of Adolescent Medicine. Clinical interview was used as a diagnostic tool. They were then re-evaluated by the Department of Child and Adolescent Psychiatry where the diagnosis was confirmed.

Patients were hospitalized if they met one or more of the following criteria for admission to the inpatient ward: severe malnutrition $(<75 \%$ expected body weight) and no successful follow-up as an outpatient, vital sign instability (bradycardia $<45 /$ beats per minute, hypotension $<80 / 50 \mathrm{~mm} \mathrm{Hg}$, hypothermia $\left.<36^{\circ} \mathrm{C}\right)$, orthostatic changes in pulse $(>35$ $\mathrm{bpm}$ ) and blood pressure, severe dehydration, cardiac arrhythmias, electrolyte disturbances, acute food refusal, uncontrollable binging and purging. Our hospitalization criteria were based on the guidelines published by the Society for Adolescent Health and Medicine ${ }^{3}$.

Patients were accepted to a general pediatric ward for adolescent age group. A team consisting of an Adolescent Medicine specialist, a Child and Adolescent Psychiatrist and a dietician that has specialized in ED provides care for in-patient treatment; on the ward a pediatric consultant with 3 pediatric residents (two first, one third year) and 2-3 interns $\left(6^{\text {th }}\right.$ year medical students) are on duty. Nursing professionals on the ward have pediatric but not psychiatric training.

Study design

All information was obtained by a retrospective chart review. Type of ED, demographic factors such as age and sex, anthropometric measurements such as weight and height at admittance and the presence of an electrolyte imbalance was recorded. Duration of illness and total weight loss were obtained from the patient's history. Detailed dietary intake and calories at admittance was recorded by a pediatric dietician from a diet recall, since the symptoms began. Body mass index (BMI) was calculated (weight in kilograms divided by the square of height in meters). A detailed menstrual history including weight at last menses was obtained.

Baseline weight was defined as the weight obtained at 07:00 am on the first full day after admission. For each subject, change in weight was calculated for each day of hospitalization. Length of stay was defined as the number of full days from admission to discharge. For patients with two hospital admissions, first admission data was included in the descriptive statistics. Details about the second admission were given in the results section. The Research Ethics Board of Hacettepe University approved this study.

\section{Medical and nutritional rehabilitation}

The initial goal of inpatient treatment was medical stabilization, such as stabilization of vital signs and restoration of electrolyte balance and homeostasis, which was ensured through nutritional rehabilitation. If the patients were medically unstable, they were placed on bed rest until vital signs improve. All patients were followed with 24-hour cardiac monitoring for bradycardia or arrhythmias until stable. Orthostatic changes of blood pressure and pulse were monitored every morning and vital signs were continuously evaluated during the day at hourly intervals. 
Nutritional rehabilitation was started as soon as the patient was hospitalized and initial calories served depended on the type of ED and also the patient's diet history and calorie intake before hospitalization. If the patient was consuming a calorie less than $750 \mathrm{kcal} /$ day, then 750 $\mathrm{kcal}$ divided into 3 meals was started. If the patient was consuming greater than $750 \mathrm{kcal}$, then $250 \mathrm{kcal}$ was added to the consumption and depending on the calories divided into 3 main meals and 1-3 snacks. For patients with $\mathrm{AN}$, the meal plan was managed according to daily weight gain, (which was measured in a gown daily before breakfast, after urination) and increased $250 \mathrm{kcal}$ if weight gain was less than 200 grams/day. An inpatient weight gain criterion of maximum $1.0 \mathrm{~kg} /$ week was accepted safe to avoid complications of refeeding. Patients were followed closely for refeeding syndrome and electrolytes were obtained daily for the first 5 days and longer if necessary. Nutritional rehabilitation was started by eating regular food whenever possible. Liquid nutrition supplements per oral or by nasogastric tube feeding if they denied drinking were used for short term if necessary, such as during refusal to eat regular food.

A six-meal-a day plan (3 main meals and 3 snacks) was initiated for BN patients. The calorie amount was calculated according to daily consumption on admission.

All meals were accompanied by the residents or interns to ensure that the meal was consumed. For patients with a history of purging, the bathroom was locked for at least half an hour after each meal. All patients were on strict bed rest until medically stable.

Criteria for discharge were medical stabilization and nutritional rehabilitation, regular consumption of all meals with no reported binge eating or vomiting and an establishment of therapeutic alliance for the psychiatric followup as well as initiating weight restoration. Full recovery of weight to ideal target weight was not a criterion for discharge but an outpatient treatment goal for patients with AN. Target weight was estimated according the weight of regaining regular menses for females, growth charts for males and females who did not have menarche and additionally the median weightfor-height adjusted for age.

\section{Results}

Thirty-seven adolescents with ED met criteria for admission to the general pediatric ward of our hospital. According to diagnostic criteria for ED's (DSM IV-TR and DSM 5), 24 (64.9\%) patients were diagnosed with anorexia nervosa restricting type (AN-R), 8 (21.6\%) with anorexia nervosa binging purging type (ANBP), $3(8.1 \%)$ with BN and $2(5.4 \%)$ patients with Eating disorder otherwise not specified (EDNOS). The 2 patients with EDNOS and their parents refused inpatient admittance and

Table I. Characteristics of Inpatients with Anorexia Nervosa (AN) and Bulimia Nervosa (BN).

\begin{tabular}{lll}
\hline & AN $(\mathrm{N}: 32)$ & BN $(\mathrm{N}: 3)$ \\
Characteristics & Mean $\pm \mathrm{SD}$ (min-max) & Mean \pm SD (min-max) \\
\hline Admission age (years) & $14.6 \pm 1.8(11.3-17.8)$ & $16.3 \pm 0.5(15.6-16.6)$ \\
Age at onset of symptoms (years) & $13.8 \pm 1.4(11.0-16.7)$ & $15.0 \pm 0.8(14.2-15.8)$ \\
& $25: 7$ & $3: 0$ \\
Sex (Female:Male) & $14.9 \pm 1.9(12.6-19.7)$ & $20.4 \pm 3.9(17.4-28.8)$ \\
Admission BMI (kg/m²) & $4.7 \pm 7.6(0.1-28.1)$ & $40.3 \pm 41.3(7.9-86.7)$ \\
Admission BMI percentile & $-2.9 \pm 1.7(-6.4-0.6)$ & $-0.3 \pm 1.3(-1.4-1.1)$ \\
Admission BMI z-score & $16.3 \pm 1.9(12.6-19.7)$ & $21.3 \pm 2.5(19.4-24.1)$ \\
Discharge BMI (kg/m²) & $12.7 \pm 15.0(0.1-58.3)$ & $55.5 \pm 24.8(35.9-83.4)$ \\
Discharge BMI percentile & $-1.8 \pm 1.4(-5.6-0.2)$ & $0.2 \pm 0.7(-0.4-1.0)$ \\
Discharge BMI z-score &
\end{tabular}

BMI: body mass index; max: maximum; min: minimum; SD: standard deviation 
follow-up. A total of 35 patients were admitted. The mean age at admission was $14.79 \pm 1.75$ (min-max: 11.25-17.83) years and 7 (20\%) of the adolescents were males. The symptoms began in early or middle adolescence in all cases. The mean age of onset of symptoms was $13.92 \pm 1.43$ (min-max: 11.00-16.75) years. The mean interval between the onset of symptoms and the diagnosis was $9.95 \pm$ 9.79 (min-max: 1-54) months. Age and BMI characteristics of patients with AN and BN at the time of admittance and discharge have been given in Table I. Seventy-eight percent of the patients with AN were malnourished weighing $80 \%$ or less of their ideal body weight, at the time of admission. Mean weight lost from lifetime maximum to admission was $19.2 \pm 10.2$ (min-max: 6.0-43.0) kg. All patients except one had their premorbid weights recorded. According to the weight for height calculations; $2(6.3 \%)$ of the adolescents were morbidly obese, 7 (21.9\%) were obese, 2 (6.3\%) were overweight, 15 (46.9\%) were normal, 4 (12.5\%) were underweight, and 1 (3.1\%) was malnourished. Other than a previously obese patient becoming normal weight, the rest of the overweight and obese patients became malnourished or underweight after the onset of AN. Two of the BN patients were previously overweight, the other BN patient's weight was normal. Height below the $50^{\text {th }}$ percentile was evident in $18(56.3 \%)$ patients. None of the patients were below or equal to the $3^{\text {rd }}$ percentile for height.

Fourteen AN patients had compensatory behaviors other than vomiting, which were over exercising (n: 8), use of laxatives (n: 5), and diuretics (n: 1). None of the patients had pre-existing medical-comorbidities. One patient with AN was also diagnosed with obsessive compulsive disorder.

Indications for hospitalization have been given in Table II. A majority of patients were hospitalized due to medical instability (n: 29). Bradycardia and hypothermia were the most common physiological instabilities. Vital signs of patients at admittance have been given in Table III. Three patients were found to be severely dehydrated and one of them was diagnosed with acute renal failure at the time of admittance due to severely restricted water intake. All severely dehydrated patients were treated with intravenous fluid administration (mean rehydration period: 2.67 days) and the renal functions of the last patient also improved in two days with rehydration. Eighteen patients who were admitted due to their medical instabilities were also severely malnourished (weight was under $75^{\text {th }}$ percentile) at admission. Low triiodothyronine syndrome was present in 23 patients with AN. Low bone density, z-score lower than -2 , was detected in 4 patients via dual-energy X-ray absorptiometry. The

Table II. Indications for Hospitalization of Patients with Eating Disorders.

\begin{tabular}{ll}
\hline Indications for Hospitalization & $\mathrm{N}(\%)$ \\
\hline Vital signs & $29(82.3)$ \\
Severe bradycardia $(<45 \mathrm{bpm})$ & $16(45.7)$ \\
Hypothermia $\left(<35.6^{\circ} \mathrm{C}\right)$ & $12(34.2)$ \\
Hypotension $(<80 / 50 \mathrm{~mm} \mathrm{Hg})$ & $9(25.7)$ \\
Orthostatic changes in pulse $(>35 \mathrm{bpm})$ & $5(14.2)$ \\
Orthostatic changes in blood pressure $(>20 \mathrm{~mm} \mathrm{Hg})$ & $1(2.8)$ \\
Severe malnutrition* & $18(51.4)$ \\
Acute food refusal & $5(14.3)$ \\
Failure of outpatient treatment & $4(11.4)$ \\
Uncontrollable binging and purging & $3(8.6)$ \\
Electrolyte disturbances & $2(5.7)^{* *}$ \\
Severe dehydration & $2(5.7)$ \\
Comorbid diagnosis that interferes with the eating disorder & $1(2.8)^{* * *}$ \\
\hline
\end{tabular}

${ }^{*}$ Weight $\leq 75 \%$ average body weight for age, sex, and height; ${ }^{* *}$ hypokalemia; ${ }^{* * *}$ obsessive-compulsive disorder 
mean period of admittance was $26.4 \pm 11.9$ (min- max: 9-61) days for AN patients and 23.7 \pm 15.03 (min-max: 19-29) for BN patients. Mean period of time from admittance to medical stabilization was $6.04 \pm 4.79$ (minmax: 1-21) days.

The mean calorie that the patients with AN-R had been consuming before they applied to our clinic was $607 \pm 333 \mathrm{kkal}$ (min-max: $0-1,500 \mathrm{kcal}$ ) and for AN-BP type this was $1038 \pm 906$ (min-max: 500-3,000). Mean calorie prescribed during hospital course was as follows: $974 \pm 333$ (min-max:750-1,750) $\mathrm{kcal}$ at admittance, 1,616 \pm 371 (min-max: $1,000-2,250) \mathrm{kcal}$ at a week after admittance and 2,358 \pm 605 (min-max:750-3,500) kcal at discharge. Although daily calorie intake was started low and increased slowly according to weight gain for inpatients, hypophosphatemia occurred in 2 patients during refeeding. The initial phosphate level was $3.74 \mathrm{mg} / \mathrm{dl}$ and $3.16 \mathrm{mg} / \mathrm{dl}$ and dropped to $2.5 \mathrm{mg} / \mathrm{dl}$ on day 2 with $1,000 \mathrm{kcal} /$ day and $1.96 \mathrm{mg} / \mathrm{dl}$ on day 3 with $1250 \mathrm{kcal}$ respectively. Replacement with oral neutral phosphate was given at $2 \mathrm{cc} / \mathrm{kg}$ for 3 days for both.

During the hospitalization period mean weight gained was calculated as $247 \pm 1057 \mathrm{~g}$ during the first week, $1,109 \pm 1,018 \mathrm{~g}$ during the second week, $1,143 \pm 793 \mathrm{~g}$ during the third week and 1,422 $\pm 1,199$ during the fourth week. Mean total weight gained during the whole hospitalization period was 3,950 \pm $3,524 \mathrm{~g}$.

Twenty patients with AN (62.5\%) succeeded to reach their target weight and the mean interval between the date of admittance and the date of reaching the target weight was $6.3 \pm 4.3$ (min-max: 2-18) months. Only one patient with AN had been able to reach her target weight at the period of discharge and this was probably because her hospital stay was the longest at 61 days.

Among the 35 patients 17 completed the outpatient follow-up in the Adolescent Medicine Clinic as they reached and maintained their target weights and are now only followed yearly. Mean follow-up period of these patients after discharge was $16.1 \pm 9.8$ (min-max: 4.0-35.0) months and mean interval between the date of admittance and the date of reaching the target weight was $4.9 \pm 3.0$ (min-max:1.0-13.0) months. Five patients discontinued their outpatient treatment before reaching their target weight and mean follow-up time of these patients was $7.8 \pm 5.5$ (3.0-13.0) months. Thirteen patients that are still being closely followed at the division have been followed for a mean period of $8.0 \pm 7.0$ (min-max: 2.0-24.0) months. Among these patients 6 have reached their target weight and the mean interval time from their admittance to the date of reaching the target weight was $8.2 \pm 6.6$ (min-max: 1.0-18.0) months.

Of the 25 female patients 5 patients with AN were premenarchal, and the remaining 20 had lost menstrual cycles. The mean duration of amenorrhea was $6.63 \pm 4.31$ (3-18) months. The mean time for the onset of menstrual cycles after admission was $7.1 \pm 6.15$ (3-22) months in 11 patients. Among the remaining 9 amenorrheic patients, 5 had rejected to continue their outpatient follow-up and the remaining 4 patients are still being followed. All premenarchal patients had reached their target weight and four of them completed the outpatient treatment.

During their hospital stay $91.4 \%$ of patients were prescribed psychopharmacotherapy. Medications used were olanzapine and/or fluoxetine. Nasogastric tube feeding was indicated in $4(12.5 \%)$ patients with $\mathrm{AN}$ and

Table III. Vital Signs of Anorexia Nervosa Patients at Admittance.

\begin{tabular}{ccccccc}
\hline & $\begin{array}{c}\text { HR } \\
(\mathrm{bpm})\end{array}$ & $\begin{array}{c}\text { Orthostatic } \\
\text { HR change } \\
(\mathrm{bpm})\end{array}$ & $\begin{array}{c}\text { Body } \\
\text { temperature } \\
\left({ }^{\circ} \mathrm{C}\right)\end{array}$ & $\begin{array}{c}\text { Systolic BP } \\
(\mathrm{mm} \mathrm{Hg})\end{array}$ & $\begin{array}{c}\text { Diastolic BP } \\
(\mathrm{mm} \mathrm{Hg})\end{array}$ & $\begin{array}{c}\text { Orthostatic } \\
\text { BP change } \\
(\mathrm{mm} H \mathrm{Hg})\end{array}$ \\
\hline Mean $\pm \mathrm{SD}$ & $\begin{array}{c}60.9 \pm \\
16.3\end{array}$ & $\begin{array}{c}13.6 \pm \\
8.7\end{array}$ & $35.9 \pm 0.6$ & $82.1 \pm$ & $49.7 \pm 8.9$ & $5.2 \pm 5.6$ \\
Minimum & 40.0 & 0.0 & 35.3 & 60.0 & 40.0 & 0.00 \\
Maximum & 120.0 & 30.0 & 36.8 & 110.0 & 65.0 & 20.00 \\
\hline
\end{tabular}

BP: blood pressure; bpm: beat per minute; HR: heart rate; SD: standard deviation 
drip infusion was used in one patient. The mean period of nasogastric tube feeding was $2.5 \pm$ 1.3 days (min-max:1-4). Enteral formulas were given to $12(37.5 \%)$ patients with AN. During hospitalization one patient developed peripheral edema as a complication, which was thought to be related to protein over consumption.

All except two met the discharge criteria for completion of treatment as an inpatient. One patient dropped out of the program on day 21 of her hospitalization, after the decision of her staying in the pediatric ward without a companion was rejected by the mother. She came back 4 months later, was re-admitted and this time completed the course with a hospitalization period of 25 days. The second patient was also discharged 2 days after her hospitalization against medical advice. After 36 days of outpatient follow-up she was readmitted for a course of 21 days.

After completing treatment as an inpatient, 2 patients were re-admitted. One was a patient with AN-BP who was admitted due to acute food refusal and stayed in the pediatric ward for 32 days. One month after her discharge she was admitted to the child intensive care unit due to severe bradycardia and hypotension. After three days her vital signs became stable and because her binge and vomiting attacks were uncontrollable and she was now over 18 years of age, she was referred to the psychiatric ward for adults of the same hospital. Total hospitalization period of her second admission was 46 days. The other patient's diagnosis was AN-R and she refused follow-up as an outpatient after discharge and we later found out that she had been readmitted to another hospital.

\section{Discussion}

The aim of this study was to evaluate the outcome of patients with an ED needing hospitalization on a general pediatric ward. Services for treating eating disorders range on a continuum of intensity from inpatient hospitalization at specialized ED facilities to residential care to partial hospitalization programs to varying levels of outpatient treatment ${ }^{2}$. Decisions about the best treatment setting in which to manage an adolescent with an ED depends on the nature of the disorder, medical and psychological complications, availability of facilities and insurance issues ${ }^{6}$.
Evidence shows that the outcome of adolescents with eating disorders treated in specialized eating disorder facilities where staff has experience and expertise in treating eating disorders is superior and evidence that structured programs of care improve outcomes has been shown for a number of different disease areas not only ED's ${ }^{7}$. This being said, major restrictions in selecting the most appropriate treatment setting include geographic proximity or lack of specialist centers that limit access, deficiency of data for comparing cost and effectiveness of different settings and inadequate insurance coverage, that limit access to effective care. So what happens when specialized centers are not available?

At our hospital patients are hospitalized on a general pediatric ward but followed by an ED team consisting primarily of an adolescent medicine specialist, child and adolescent psychiatrist and a dietician. As can be seen from the results of our study, hospitalization of our ED patients was managed successfully and we do believe this approach to have certain advantages that should not be overlooked.

A majority of these patients are extremely malnourished, and therefore compromised, with severe medical complications. When we evaluate the guidelines for hospitalization of an adolescent for an $\mathrm{ED}^{3}$, the majority of these recommendations are medical. If these patients are followed on psychiatric wards where pediatricians are not always on call this can also lead to major problems as psychiatrists and medical staff in psychiatric wards may not have sufficient training to handle severe medical complications and emergency medical equipment is not sufficiently accessible for dealing with a sudden change in the condition of a severely ill patient.

Additionally, more interventional procedures such as the need for a nasogastric tube placement are also placed by pediatricians or pediatric nurses on our wards this is also another advantage as on a psychiatric ward trained staff may not always be available.

Adult ED patients are commonly hospitalized on psychiatric wards and followed solely by psychiatrists but the decision of where to treat an adolescent ED patient should be evaluated carefully. The presence of an adolescent medicine specialist or pediatrician 
is of extreme importance when the patient is an adolescent as a psychiatrist may not be aware of the unique physiologic, metabolic, and developmental differences between adolescents and adults.

In our country, the number of child psychiatry inpatient wards is also limited. The only other option for an ED patient is the adult psychiatric ward in our institution. Being amongst adults with chronic psychiatric illnesses can sometimes be very traumatic for the adolescent as we have experienced with some of our own patients.

There are three main parts to the goals of inpatient treatment, first is medical stabilization, second nutritional rehabilitation and third achievement of goal weight ${ }^{8}$. A major limitation to our inpatient setting was that we were not able to complete the third part of treatment. Studies show that one of the most important findings in predicting the prognosis of an ED patient is weight at discharge ${ }^{9}$. The main reason for this is that the hospital is a tertiary care hospital which receives referrals from all over the country, the need to hospitalize patients more often exceeds the capacity of our inpatient wards so that after our patients are medically stable and nutritional rehabilitation has been achieved we are asked to discharge them in order to make space for other patients needing critical care. When we evaluate the literature there is conflicting evidence to guide as to what the most appropriate length of stay is and studies have shown the length of hospitalization for adolescents with AN ranged greatly ${ }^{4}$. A trial by Madden et al. 10 compared brief hospitalization for medical stabilization with prolonged hospitalization for weight normalization. In this trial the authors evaluated differences in outcome as measured by changes in weight and ED pathology which they measured by using the Eating Disorder Examination Scale ${ }^{11}$. No significant differences were observed for each group and they emphasized the significance on costs savings when adolescents were hospitalized for a shorter length. Whereas in another study by Steinhausen et al. ${ }^{12}$, they showed that hospitalization of near to normal weight improves outcomes and decreases the need for hospitalization over the course of the illness. A study evaluating the variations in admission practices for adolescents with AN in North American showed that the length of stay ranged from 2-90 days. It was argued that the variations were due to whether the physician worked in a setting were insurance was an issue ${ }^{4}$. In our study the mean period of admittance was $26.4 \pm 11.9$ days (min- max: 9-61) as insurance is not a factor in health care decisions in Turkey this did not affect our decision to discharge.

We are also confronted with other problems while following ED patients on general pediatric wards. A major advantage to a specialized ED ward is that the patient's daily activity is structured so that the patient has a full day program. But on our general pediatric wards, we are not able to offer such facilities and our patients spend a majority of their time just watching television, reading or searching on the web. Lack of things to do gives them time to dwell on their ED problem which is something we do not want.

An important part of the psychiatric inpatient treatment is that of separation and individualism. Studies show that certain patients with AN have been unable to psychologically separate from their parents which results in a failure to attain any stable sense of their own body ${ }^{13}$. This can be achieved during hospitalization by separating the parents and adolescent but again this can be difficult in a pediatric ward when every other patient is allowed the companionship of one of their parents.

Another problem is that due to hospital policies, we are not able to offer the patient a trial stay home period or to make the changeover smoother. We have no residential day care treatment centers. Having no system of transition can make it hard for the patient to adapt. Additional problems are that the nurses on the wards are pediatric nurses and have no formal psychiatric training. Each hospital room has 2 beds which means we are not able to isolate patients which may be necessary if for example the patient also has suicidal ideation. We have had the parents of the child in the bed next to our patient try to feed or encourage them to eat. Monitoring intake is usually undertaken by the interns but on a busy ward this too can sometimes be overlooked.

In this patient population, the primary goal of physiologic stabilization of vital signs through 
nutritional rehabilitation and initiating weight restoration was obtained in all our patients within 3 weeks of hospitalization, the mean being approximately 6 days.

While medically stabilizing, and initiating weight gain this must be balanced against the risk of refeeding syndrome, which is the potentially fatal metabolic disturbance that occur as a result of the reinstitution of nutrition to the ED patient ${ }^{14,15}$. The main risk for the occurrence of refeeding syndrome in patients with $\mathrm{AN}$ is related with the caloric level of the diet. For this reason, previously AN refeeding protocols generally began at lower calories increasing calorie intake gradually ${ }^{16}$. Recently inpatient programs have begun to refeed with higher calories ${ }^{17}$. The argument behind the 'treating faster and higher' lies behind the evidence that demonstrate that patients discharged at a low weight are rehospitalized at a greater rate than those who achieve their treatment goal weight within the hospital ${ }^{12,18}$.

At our institute we basically follow a 'start low, increase slow' protocol but in a very individualized manner, with no constant standard beginning protocol. Thus, this sometimes but not always means "start low" depending on the diet history of the patient. However, "increase slow" applies to most patients depending on the daily weight gain follow-ups which is mostly the consensus in the literature. The subjects admitted to our hospital were started on a mean calorie of $974 \mathrm{kcal}$ and discharged with a mean calorie of $2,360 \mathrm{kcal}$.

We prefer to be on the safer side of refeeding on busy pediatric wards and our more conservative strategy was successful as none of our subjects developed refeeding syndrome, only 2 required phosphate supplementation, and a mean weight gain of $4 \mathrm{~kg}$ were achieved.

The debate concerning prophylactic phosphate to prevent refeeding syndrome continues. While some authors advise that daily phosphate supplementation should be given to all hospitalized patients during the initial hospitalization period ${ }^{19}$, others like us recommend close early monitoring and supplementation only when phosphate levels begin to decline ${ }^{16}$. Hypophosphatemia occurred on the second and third day of admission when receiving 1,000 and $1,250 \mathrm{kcal}$ respectively in 2 of our patients. There were no adverse events or clinical symptoms requiring any extra medical intervention except for oral neutral phosphate supplementation. Additionally, authors of protocols that opt to treat prophylactically have still closely monitored their patients for the first week which means patients will still have blood drawn daily.

In conclusion, the results of this study show that patients with ED can successfully be followed on general pediatric wards with the appropriate team. We do not suggest that this approach can replace special ED units and although the conditions are not ideal, the results of this approach are encouraging and could have implications at centers with no inpatient wards specific for ED patients.

\section{REFERENCES}

1. Golden NH, Katzman DK, Sawyer SM, et al. Update on the medical management of eating disorders in adolescents. J Adolesc Health 2015; 56: 370-375.

2. Katzman DK, Kanbur NO, Steinegger CM. Chapter 16: Medical Screening and Management of Eating Disorders in Adolescents. In: Agras WS, (ed). The Oxford Handbook of Eating Disorders. New York: Oxford University, Press, Inc. 2010: 267-272.

3. Kreipe RE, Golden NH, Katzman DK, et al. Eating disorders in adolescents. Position Paper of the Society for Adolescent Medicine. J Adolesc Health 2003; 16: 496-503.

4. Schwartz BI, Mansbach JM, Marion JG, Katzman DK, Forman SF. Variations in admission practices for adolescents with anorexia nervosa: A North American sample. J Adolesc Health 2008; 43: 425-431.

5. Silber TJ, Delaney D, Samuels J. Anorexia nervosa: hospitalization on adolescent medicine units and third-party payments. J Adolesc Health Care. 1989; 10: $122-125$.

6. Madden S, Hay P, Touyz S. Systematic review of evidence for different treatment settings in anorexia nervosa. W J Psych 2015; 5: 147-157.

7. Rous E, Ranote S, Elvins R, Bhatt A. A manualised in-patient programme for young people with anorexia based on NICE guidance. Psychiatric Bulletin 2009; 33: 135-137.

8. Katzman DK. Medical complications in adolescents with anorexia nervosa: A review of the literature. Int J Eat Disord 2005; 37: 52-59.

9. Lock J, Litt I. What predicts maintenance of weight for adolescents medically hospitalized for anorexia nervosa? Eat Disord 2003; 11: 1-7.

10. Madden S, Miskovic-Wheatley J, Wallis A, et al. A randomized controlled trial of in-patient treatment for anorexia nervosa in medically unstable adolescents. Psychol Med 2015; 45: 415-427. 
11. Cooper Z, Cooper PJ, Fairburn CG. The validity of the eating disorder examination and its subscales. $\mathrm{Br}$ J Psychiatry 1989; 154: 807-812.

12. Steinhausen HC, Grigoroiu-Serbanescu M, Boyadjieva S, Neumärker KJ, Winkler Metzke C. Course and predictors of rehospitalization in adolescent anorexia nervosa in a multisite study. Int J Eat Disord 2008; 41: 29-36.

13. Akgül S, Akdemir D, Kara M, Derman O, Çetin FÇ, Kanbur N. The understanding of risk factors for eating disorders in male adolescents. Int J Adolesc Med Health 2016; 28: 97-105.

14. Kohn MR, Golden NH, Shenker IR. Cardiac arrest and delirium: Presentations of the refeeding syndrome in severely malnourished adolescents with anorexia nervosa. J Adolesc Health 1998; 22: 239-243.
15. Solomon S, Kirby D. The refeeding syndrome: A review. JPEN J Parenter Enteral Nutr 1990; 14: 90-97.

16. Ornstein RM, Golden NH, Jacobson MS, Shenker IR Hypophosphatemia during nutritional rehabilitation in anorexia nervosa: Implications for refeeding and monitoring. J Adolesc Health 2003; 32: 83-88.

17. Garber AK, Mauldin K, Michihata N, Buckelew SM, Shafer M-A, Moscicki A-B. Higher calorie diets increase rate of weight gain and shorten hospital stay in hospitalized adolescents with anorexia nervosa. J Adolesc Health 2013; 53: 579-584.

18. Baran SA, Weltzin TE, Kaye WH. Low discharge weight and outcome in anorexia nervosa. Am J Psychiatry 1995; 152: 1070-1072.

19. Leitner M, Burstein B, Agostino H. Prophylactic phosphate supplementation for the inpatient treatment of restrictive eating disorders. J Adolesc Health 2016; 58: $616-620$. 